\title{
An adaptive liquid lens with radial interdigitated electrode
}

\author{
Miao $X u^{1}$, Daming $\mathrm{Xu}^{2}$, Hongwen Ren ${ }^{1}$, II-Sou Yoo ${ }^{1}$ and Qiong-Hua Wang ${ }^{3}$ \\ ${ }^{1}$ BK Plus Haptic Polymer Composite Research Team, The Department of Polymer Nano Science and \\ Technology, Chonbuk National University, Jeonju, 561-756, Republic of Korea \\ ${ }^{2}$ CREOL, The College of Optics and Photonics, University of Central Florida, Orlando, FL 32816, USA \\ ${ }^{3}$ School of Electronics and Information Engineering, Sichuan University, Chengdu 610065, People's \\ Republic of China
}

E-mail: hongwen@jbnu.ac.kr

Received 19 May 2014, revised 15 July 2014

Accepted for publication 28 July 2014

Published 4 September 2014

\begin{abstract}
A newly patterned electrode is designed for a dielectric liquid lens. The electrode has a radial interdigitated structure which can provide a symmetrical-nonuniform fringing field. This electric field can effectively deform the shape of a dielectric liquid droplet in radial direction, causing the focal length of the liquid droplet to change. For a lens using glycerol as the droplet and optical oil (SL-5267) as the surrounding medium, its focal length $(f)$ can be tuned in the range of $\sim-8.28 \leqslant f \leqslant \sim-4.4 \mathrm{~mm}$, when the applied voltage is changed from 0 to $120 \mathrm{~V}_{\text {rms. }}$. In contrast to previous approaches, the liquid lens with radial interdigitated electrode has the advantages of scalable aperture size, wide variability of focal length, and good optical characteristics.

Moreover, the driving voltage is insensitive to the size of the droplet.
\end{abstract}

Keywords: adaptive lens, dielectrophoretic force, tunable focus, imaging

(Some figures may appear in colour only in the online journal)

\section{Introduction}

Adaptive-focus liquid lenses have potential applications in mini-camera module, image surveillance, target tracking, and other portable electronic devices. Various approaches have been demonstrated for preparing these lenses. Traditional methods can be classified into pneumatic pumping [1-4], stimuli-response hydrogel [5], acoustic [6], ferrofluidic piston [7-9], electrically mechanical actuation [10-13], electrowetting [14-19], and dielectrophoretic effect [20-27]. Each approach has its own merits and demerits. Among them, electrowetting and dielectrophoretic effect are the most attractive approaches because of direct voltage actuation with low power consumption. These two types of lenses have similar features, e.g., two immiscible liquids are employed in the lens cell; one liquid forms a droplet on one substrate surface and the other liquid fills the outer space of the droplet. Due to the geometric symmetry, the droplet exhibits a lens character. By applying an external voltage to the liquids, the shape of the droplet can be deformed, causing the focal length of the lens to change. For an electrowetting lens, one liquid should be salty water, and the shape of the droplet is deformed by the generated electrostatic force. As a comparison, the two liquids used for a dielectric lens should be insulator but with different dielectric constants. When an electric field is applied to the liquids, the shape of the droplet is deformed by the generated dielectric force. Different from an electrowetting lens, a dielectric liquid lens may not employ water as the liquid. Therefore, some dielectric liquids with good physical properties can be chosen for improving the lens characteristics without the issues of evaporation and microbubble producing.

To deform the shape of a dielectric liquid lens, an inhomogeneous electric field is required. Such an inhomogeneous electric field in a liquid lens cell can be obtained by two methods. One is to apply a voltage across both substrates [23]. If the electrode on both substrates is continuously flat without patterning, a non-uniform electric field is obtained on the surface of the liquid droplet. Because the size of the droplet is dependent on the cell gap, a larger droplet needs a thicker cell gap. As a result, a higher voltage is required to deform the shape of a larger droplet. The other way is to use a 


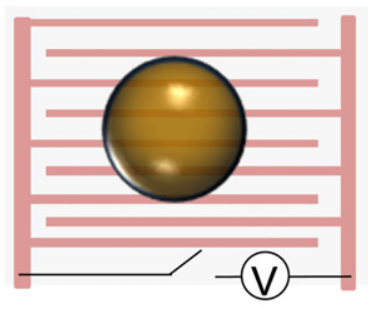

(a)
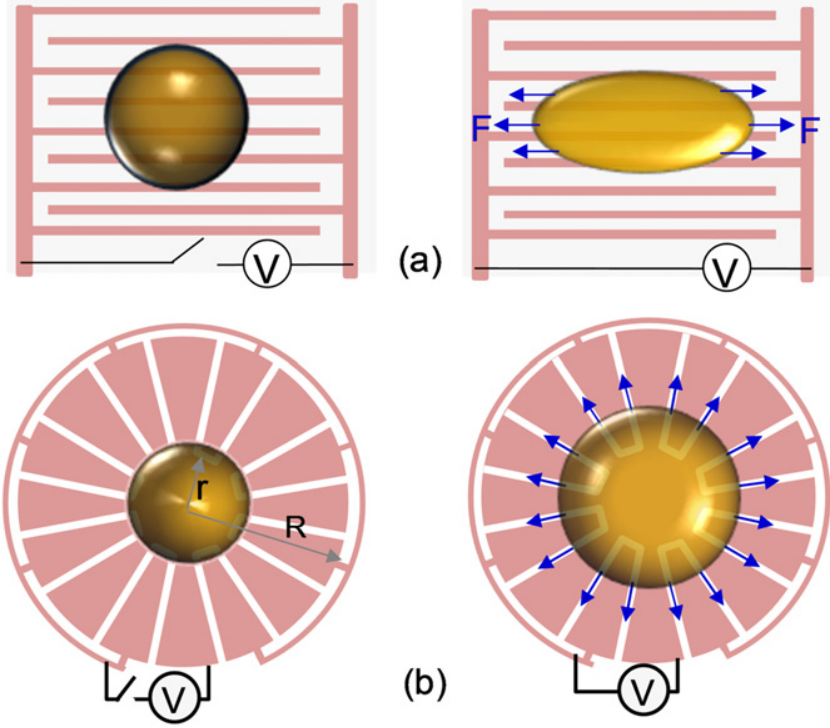

Figure 1. Mechanism of stretching the surface of a liquid droplet with two different ITO patterns: (a) parallel-interdigitated stripes, (b) radial-interdigitated stripes.

fringing field to deform the shape of a liquid droplet. The fringing field is provided by a zone-patterned electrode on one substrate [22]. Since the voltage is independent on the cell gap, the aperture of the lens is scalable. Because the zonepatterned electrode provides a discontinuous fringing field in radial direction, the shape of the liquid droplet could not be effectively deformed even though the driving voltage is high.

In this paper, we proposed a newly patterned electrode for a dielectric liquid lens. The electrode has a radial-interdigitated structure. When an external voltage is applied to this electrode, a continuously inhomogeneous electric field is generated. This field can deform the shape of a liquid droplet in radial direction, causing its focal length to change efficiently. In contrast to previous approaches, our liquid lens with the radial-interdigitated electrode has the advantages of scalable aperture size, large focal-length tunability, and good optical performance. Moreover, the driving voltage is insensitive to the gap of the lens cell.

\section{Operation mechanism and device structure}

When a dielectric object is placed in an inhomogeneous electric field, the object is subjected to a dielectric force. The force can pull the object to move in the direction towards higher electric field. This effect is called dielectrophoresis (DEP). Except for cell separation and particle manipulation [28, 29], DEP has been widely used to actuate dielectric liquid lenses. Different from conventional DEP devices, a liquid lens has special requirements about the generated electric field: inhomogeneous intensity and circularly symmetrical distribution. To generate an inhomogeneous electric field, an interdigitated electrode is desirable. Figure 1 shows the operation mechanism of a dielectric liquid droplet whose surface can be deformed by a fringing field. A dielectric droplet is dripped on the indium tin oxide (ITO) electrode. In the voltage-off state, the droplet presents a spherical shape with the lowest surface-to-volume ratio, as shown in the left side of figure 1(a). When a voltage is applied to the electrode, a fringing field is generated. Near the edges of each ITO stripe the gradient of the electric field is the highest. The molecules at the edge of the droplet experience the highest dielectric force. The dielectric force is expressed by [30]

$$
f=-\frac{\varepsilon_{0}}{2}\left(\varepsilon_{1}-\varepsilon_{2}\right) \nabla E^{2}
$$

where $\varepsilon_{0}$ is the permittivity of free space, $\varepsilon_{1}$ and $\varepsilon_{2}$ are dielectric constants of the droplet and the surrounded medium, respectively. $E$ denotes the electric field. If $\varepsilon_{1}>\varepsilon_{2}$, then these molecules are pulled to move towards the region with higher electric field. Due to the cohesive force between the adjacent molecules and the adhesive force between the droplet and the substrate, the dielectric force can stretch the surface of the droplet. Along the ITO stripes, the fringing field is continuous, while in the direction perpendicular to the ITO stripes, the electric field is discontinuous. Due to this reason, the droplet is stretched along the electrode direction, as the right side of figure 1(a) depicts [20, 21, 31]. This droplet is not suitable as a lens because of the distorted shape.

To efficiently deform a liquid droplet, the electrode can be designed with a radial-interdigitated pattern, as illustrated in the left side of figure 1(b). Each ITO strip presents a fan shape and the adjacent strips are separated with a gap. The radius of the center area without ITO is $r$ and the radius of ITO electrode from the center to the border is $R(\gg r)$. The ITO stripes are circularly symmetric. In the voltage-off state, a dielectric droplet (L-1) is placed at the center area with the smallest surface-to-volume ratio. When a voltage is applied to the electrode, the generated fringing field between each adjacent ITO stripes is continuous in radial direction. The droplet is subjected to a dielectric force in radial direction, causing the shape of the droplet to change, as illustrated in the right side of figure 1(b). Due to its spherical shape, the droplet still exhibits the lens character but with a varied focal length. Except the fluctuation surface of the droplet on the edge, the surface of the droplet is very smooth because of the surface tension. Similar to the liquid lenses with zone-patterned electrode, the optical performance of the lens shown in figure 1(b) will not be affected by the generated fringing field.

To theoretically describe the fringing field, we simulated the electric field distribution based on the ITO pattern shown in figure 1(b). The simulation has been carried out by commercial simulation software TechWiz (Sanayi System). We computed the potential distribution by solving the Poisson equation and then the distribution of electric field in the media. In the simulation, we divided the cell into multiple layers. The thickness of each layer is $0.1 \mu \mathrm{m}$. The mesh size on the horizontal direction is $0.05 \mu \mathrm{m}$. After obtaining the electric field by TechWiz, we plotted the field distribution figures in Matlab. In this structure, the gap of adjacent ITO stripes is $20 \mu \mathrm{m}$, and the width of each ITO stripe is linearly increased with the narrowest width of the ITO strip being $20 \mu \mathrm{m}$. To simplify the calculation, a $x, y, z$ coordinate system 


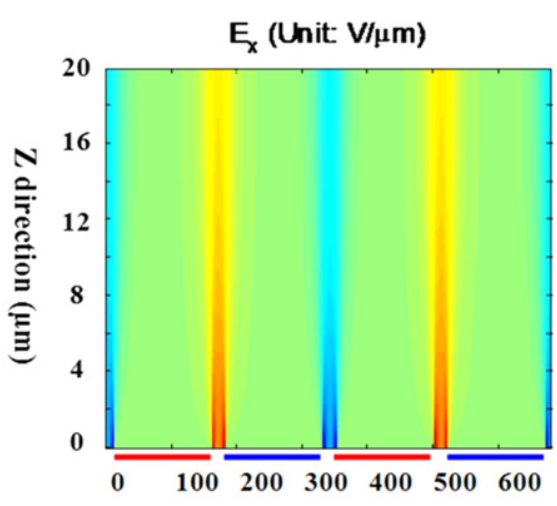

$E_{x}($ Unit: $V / \mu m)$

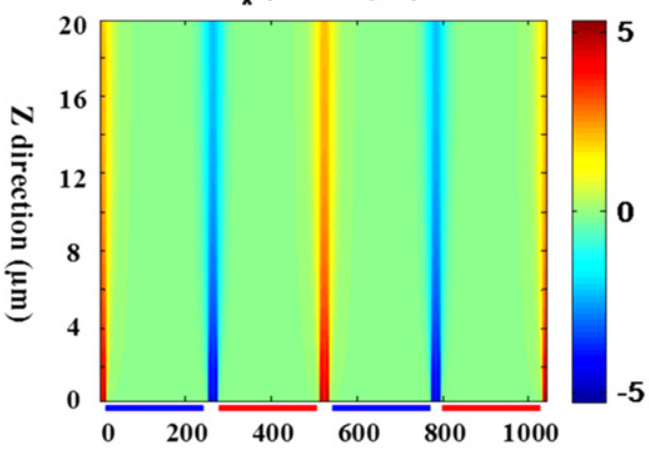

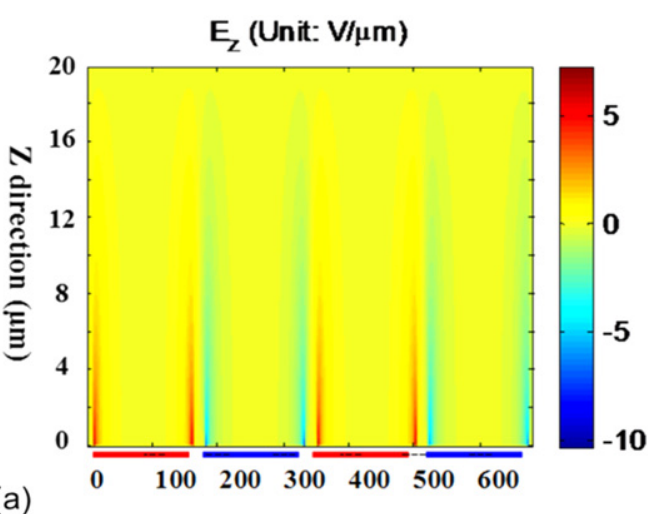

(a)

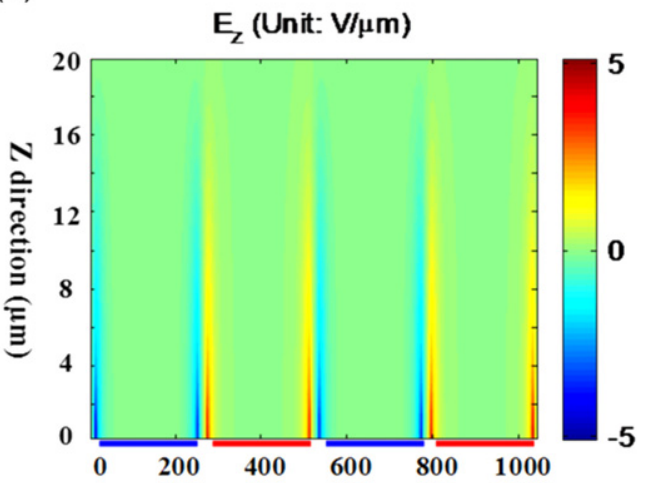

(b)

Figure 2. Electric field distribution along $x$ - and $z$-direction with $20 \mu \mathrm{m}$ ITO gap. The applied voltage is $100 \mathrm{~V}_{\text {rms }}$. The width of the ITO stripe is (a) $150 \mu \mathrm{m}$ and (b) $240 \mu \mathrm{m}$.

(not shown) is established in the center of the radial pattern. The $x$-axis is set perpendicular to one ITO stripe, the $y$-axis is along the ITO stripe, and the $z$-axis is vertical to the surface of the substrate. When a voltage is applied across the stripes, the electric field can be decomposed into two parts: along the $x$ direction $\left(E_{x}\right)$ and along the $z$-direction $\left(E_{z}\right)$. There is no electric field along the $y$-axis. Suppose the voltage is $V=100 \mathrm{~V}_{\mathrm{rms}}$ and the width of the ITO stripe is $150 \mu \mathrm{m}$, the simulated electric field distribution is given in figure 2(a). Along the $x$-direction near the edges of the ITO stripe, the electric field is the strongest. According to equation (1), the generated dielectric force is strongest in the radial direction. Since the ITO pattern is geometrically symmetrical, the generated dielectric force is symmetric too. For the ITO stripe with different width, the electrode provides the similar electric field distribution. Figure 2(b) shows the field distribution when the width of the ITO stripe is chosen to be $240 \mu \mathrm{m}$. Different from the results shown in figure 2(b), the density of fringing field becomes looser. To balance the loss of the electric field density, a feasible way is to decrease the gap of adjacent ITO stripes, decrease the width of the ITO strip, or both of them.

To enhance the mechanical stability, the outer space of the droplet (figure 1(b)) is filled with another immiscible liquid. The side-view structure of the lens cell is shown in figure 3(a). It consists of the bottom glass plate (with radial ITO stripes), the liquid droplet (L-1), the filled liquid (L-2), and the top glass plate. To prevent any charge from being injected into the liquids, a dielectric film (D-1) is coated on

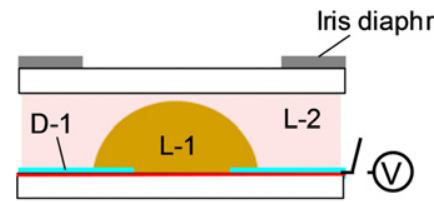

(a)

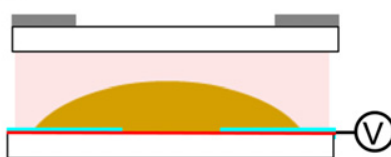

(b)
Figure 3. Side-view structure of a liquid lens using radial interdigitated electrode. (a) Relaxing state and (b) stretching state.

the electrode. When a voltage is applied to the electrode, the droplet is stretched in the radial direction, as shown in figure 3(b). Because the surface profile of the droplet is changeable, the focal length of the droplet can be tuned. When the voltage is removed, the droplet can recover to its original shape owing to the interfacial tensions. To get rid of the effect of surface undulation on the edge of the droplet and improve the contrast of imaging, an iris diaphragm is placed on the top of the cell. The opening diameter of the iris diaphragm is slightly smaller than the aperture of the droplet.

\section{Experiment}

Firstly the ITO electrode coated on one glass substrate was etched with a radial-interdigitated pattern. The gap of adjacent ITO stripes is $20 \mu \mathrm{m}$, and each ITO strip presents a fan shape. The radiuses of $r$ and $R$ are $2 \mathrm{~mm}$ and $5 \mathrm{~mm}$, respectively. Around the circular aperture there are 44 stripes. Then the ITO surface was coated with a Teflon layer $\left(\gamma_{\mathrm{T}} \sim 19\right.$ 


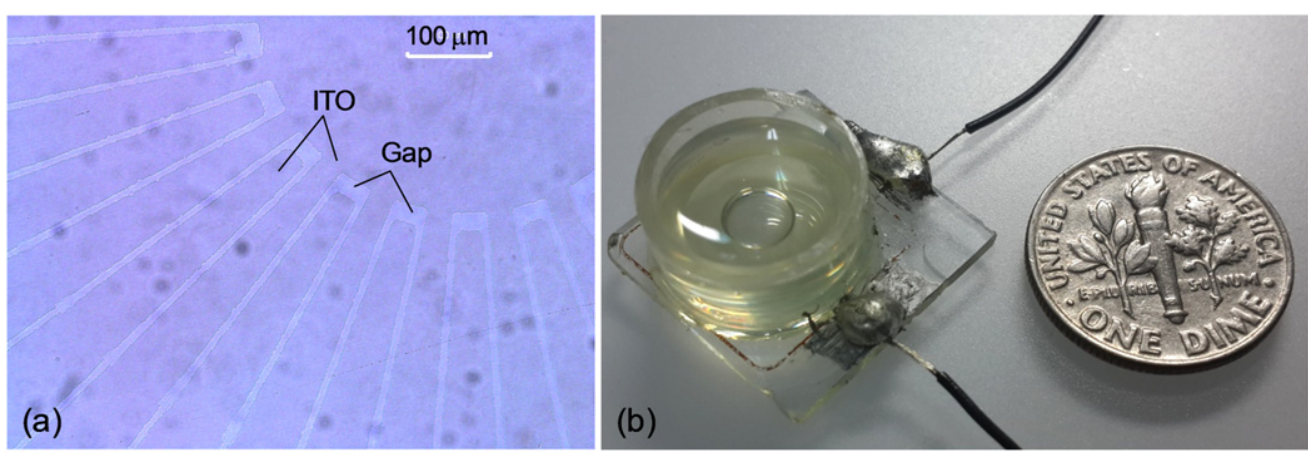

Figure 4. (a) The patterned ITO structure and (b) the top view of the assembled lens cell.

dyne $\mathrm{cm}^{-1}$ at room temperature). The Teflon layer is used as the dielectric film (D-1). To fix a liquid droplet, the Teflon layer at the center area of electrode was etched with a hole. The inner edge of the electrode does not expose in the hole. A small amount of glycerol $\left(\varepsilon_{1} \sim 47, \rho_{\mathrm{g}}=1.25 \mathrm{~g} \mathrm{~cm}^{-3}, \gamma_{\mathrm{g}} \sim 63\right.$ dyne) with high purity $(>99.5 \%)$ was dripped in the Teflon hole. The droplet contacting the bottom substrate is the aperture. The radius of the droplet aperture is $\sim 2 \mathrm{~mm}$ in the voltage-off state. A cylinder with an aperture much larger than that of the droplet is placed around the glass substrate to form a container. Then optical oil SL-5267 $\left(\varepsilon_{2} \sim 5\right.$, $\rho_{\mathrm{g}} \sim 1.26 \mathrm{~g} \mathrm{~cm}^{-3}, \gamma_{\mathrm{g}} \sim 50 \mathrm{dyn} \mathrm{cm}^{-1}$ ) was used to fill the outer space of the glycerol droplet. Finally, the two liquids were covered with another glass plate to form a lens cell. The periphery of the cell was tightly sealed using epoxy glue.

\section{Results and discussion}

Figure 4(a) shows part of the patterned electrode. The slightly dark area is covered with ITO electrode and the bright stripes are the region without ITO electrode. The size of the substrate is $1.8 \times 1.8 \mathrm{~cm}$. Figure 4 (b) shows the top-view structure of the prepared lens cell. The thickness of the cell is $\sim 7 \mathrm{~mm}$. To clearly observe the lens cell, the top glass substrate is not placed. The glycerol droplet in the center of the bottom substrate is observable. The size of the lens cell can be compared with the size of a dime.

For the glycerol droplet, its refractive index $\left(n_{1} \sim 1.47\right)$ is smaller than that of the surrounding oil $\left(n_{2} \sim 1.67\right)$, so the lens is a diverging lens. To evaluate the lens performance as its aperture is changed, a bunch of flowers was chosen as the object, as shown in figure 5(a). The object was placed under the lens cell at $\sim 20 \mathrm{~mm}$ distance. A digital camera was used to record the observed image. At $V=0$, a clear image was observed in white light circumstance, as shown in figure 5(b). The radius of the iris diaphragm placed on the top of the lens cell is $\sim 1.75 \mathrm{~mm}$. The image is virtually upright and diminished. The image begins to grow when the voltage applied to the electrode is higher than $30 \mathrm{~V}_{\mathrm{rms}}$. Figure 5(c) shows the image when $V=40 \mathrm{~V}_{\mathrm{rms}}(300 \mathrm{~Hz})$. The size of the image is magnified. This is because the curvature of the droplet is flattened, leading the power of the lens to increase. The image keeps growing when the voltage is increased. Figures 5(d) and (e) show the observed images when $V=80$ and $120 \mathrm{~V}_{\text {rms }}$, respectively. Not only the image is obviously magnified, but also the image performance is improved. To estimate the lens performance, a USAF resolution target is used to replace the flowers. In the voltage-off state, the lens could clearly resolve group 4 and element 3 , so the resolution is $\sim 20 \mathrm{lp} \mathrm{mm}^{-1}$, as shown in figure $5(\mathrm{f})$. When $V=120 \mathrm{~V}_{\mathrm{rms}}$, the resolution of the lens reaches $\sim 25 \mathrm{lp} \mathrm{mm}^{-1}$. Although the voltage is relatively high, the power consumption is only several milliwatts.

Similar to conventional glass lens, the optical performance of a liquid lens is limited by the lens aberration, diffraction limit, or both. If the lens has no aberration, then the resolution of the lens is limited by diffraction. The limiting resolution (or cutoff frequency) $R(\mathrm{C})$ is expressed by [32]

$$
R(\mathrm{C})=2(\text { N.A. }) / \lambda,
$$

where N.A. is the numerical aperture and $\lambda$ is the wavelength of light. When the lens is focused at infinity, the image-space N. $\mathrm{A}_{i}$ of the lens is

$$
\text { N.A } \approx \approx n \frac{D}{2 f}
$$

where $n$ is the refractive index of the medium in which the lens is working, $D$ is the diameter of the lens aperture, and $f$ is the focal length of the lens. From (2) and (3), we have

$$
R(\mathrm{C})=\frac{D}{f \lambda} .
$$

From (4), if the size of the aperture is fixed, then increasing the optical power of the lens $(1 / f)$ will improve the resolution of the lens. For our liquid lens, the distance of the object is much larger than the aperture of the lens, so the observed resolution of the lens satisfies (4). Because our lens is a diverging lens, its optical power increases when the curvature of the liquid interface increases. Therefore, the optical performance of our lens is improved when a higher voltage is applied to the electrode.

A diverging lens can't focus a beam to a point, so one can't measure its focal length directly. A simple way to measure its focal length is to integrate it with a convex lens, so that the focal power of the lens system is positive. A simple setup for measuring the focal length is shown in figure 6 . A collimated He-Ne laser beam $(\lambda=633 \mathrm{~nm})$ is used to illuminate the liquid lens. After converging by the imaging lens, the 

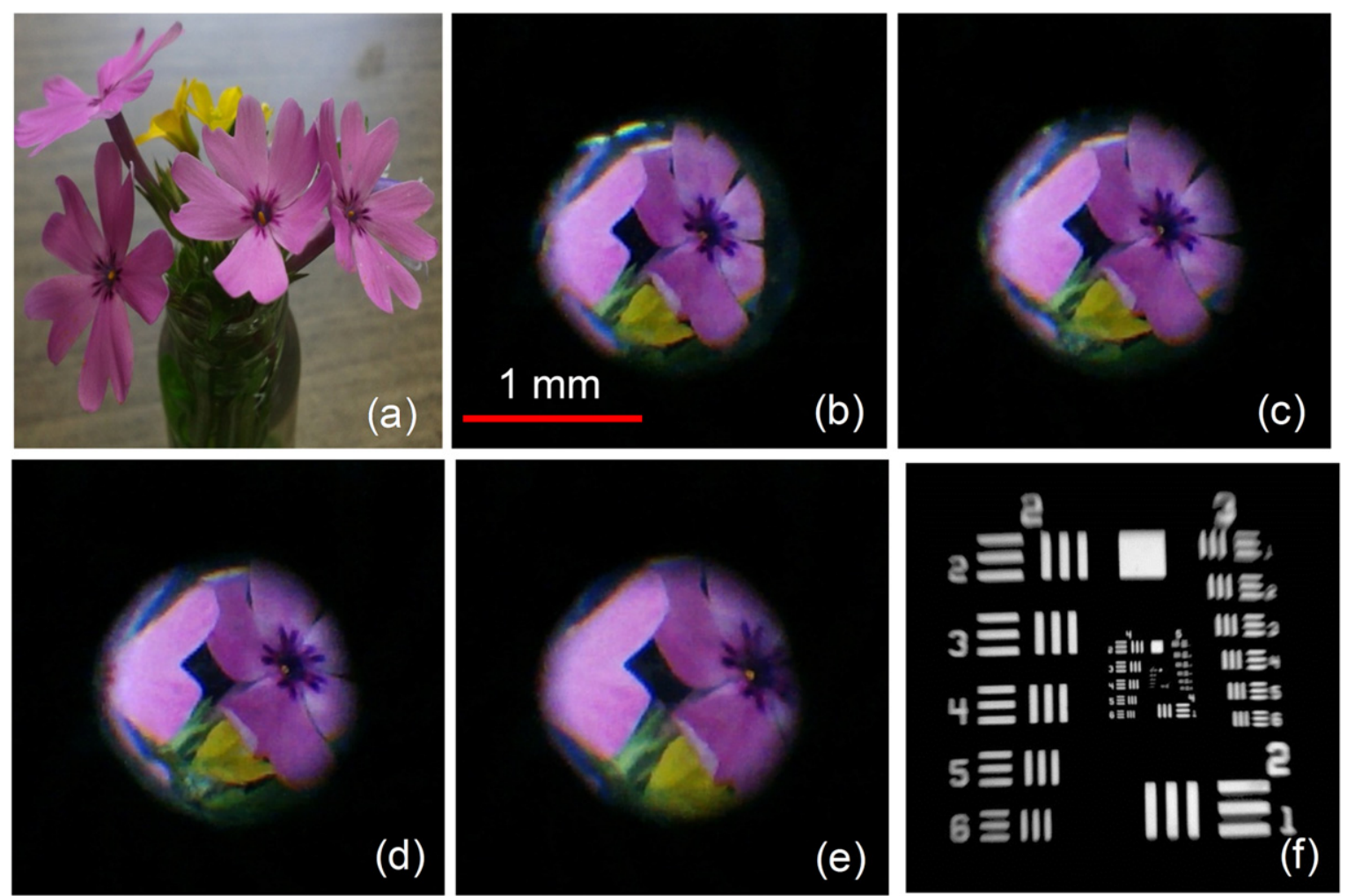

Figure 5. Object and images of the object observed through the liquid lens with different voltages. (a) Object, (b) $V=0$, (c) $V=60 \mathrm{~V}_{\text {rms }}$, (d) $V=100 \mathrm{~V}_{\text {rms }}$, (e) $V=120 \mathrm{~V}_{\text {rms }}$, and (f) resolution target at $V=0$.

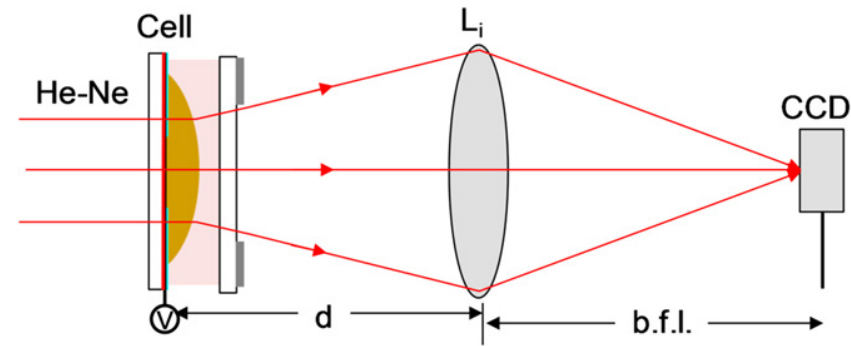

Figure 6. Setup for measuring the focal length of the liquid lens.

focused beam can be analyzed using a CCD beam profiler (BGS-USB-SP503). The distance from the focal point to the vertex of $L_{1}$ is known as the back focal length (b. f. l.). Theoretically, $b . f$. $l$. is expressed by [33]

$$
\text { b.f.l. }=\frac{f_{1}\left(d-f_{2}\right)}{d-\left(f_{1}+f_{2}\right)},
$$

where $f_{1}$ and $f_{2}$ are the focal lengths of $L_{i}$ and the lens cell, respectively. $d$ is the distance from the imaging lens to the lens cell. If $f_{1}, d$, and $b . f$. $l$. are given or measured, then $f_{2}$ can be calculated. The focal point is defined as the position with the highest light intensity. In our setup, $f_{1}=35 \mathrm{~mm}$ and $d \sim 45 \mathrm{~mm}$.

Figure 7 shows the focal length of the liquid lens as well as the droplet aperture versus the applied voltage. When the aperture of the glycerol droplet changes from $\sim 4.8 \mathrm{~mm}$ to $\sim 6.5 \mathrm{~mm}$, its focal length is tuned from $\sim-4.41 \mathrm{~mm}$ to $\sim-8.28 \mathrm{~mm}$. The bars in figure 7 denote the experimental

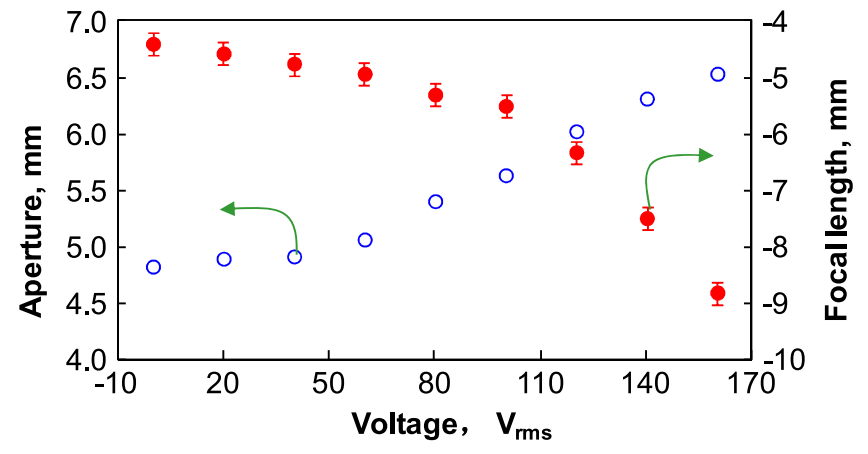

Figure 7. The dependent of focal length and droplet aperture on various voltages.

error of the focal length due to the uncertainty in determining the beam waist.

When the liquid lens is actuated for imaging, the response time of the liquid lens should be considered. It is easy to measure the response time of the liquid lens when it is impacted by a voltage pulse. In the voltage-off state, the position of the CCD is adjusted so that it can detect a lower light intensity in a defocus state. When a voltage pulse is used to impact the lens, the light is focused and it can detect a higher light intensity. The measured intensity is analyzed using a digital oscilloscope. Figure 8(a) shows the timedependent light intensity change when the liquid lens is impacted by a $100 \mathrm{~V}_{\mathrm{rms}}$ pulse. The duration of the voltage pulse is $7 \mathrm{~s}$, and the frequency of the squared pulse is $300 \mathrm{~Hz}$. It takes $\tau_{\text {rise }} \sim 6 \mathrm{~s}$ for the droplet to expand its interface to a 

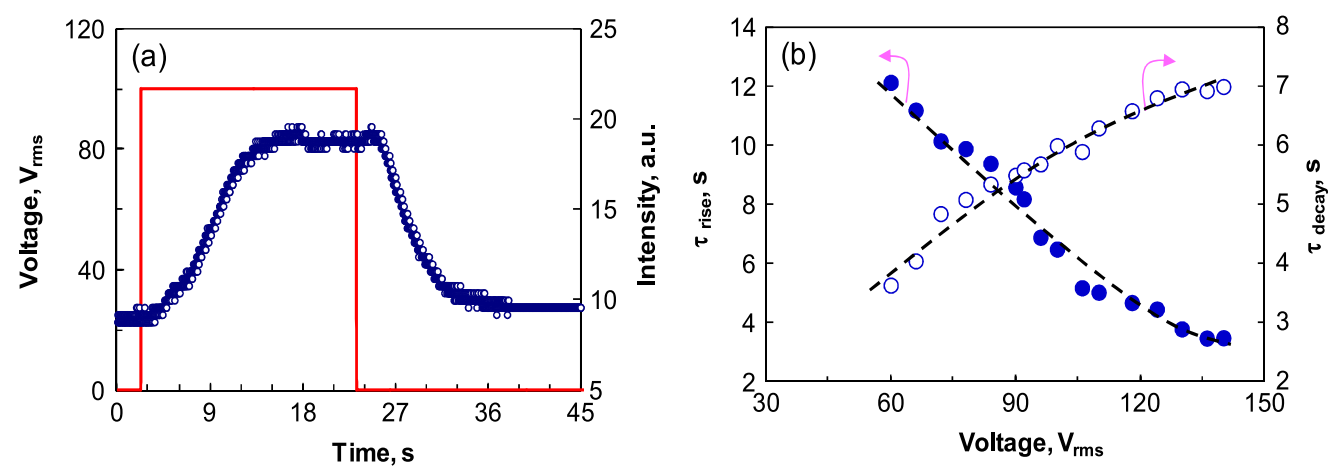

Figure 8. Dynamic response of the liquid lens impacted by (a) $100 \mathrm{~V}_{\mathrm{rms}}$ voltage pulse and (b) various voltage pulses. The frequency of the voltage is $300 \mathrm{~Hz}$.

stable state and $\tau_{\text {decay }} \sim 6.5 \mathrm{~s}$ for the droplet to recover to its original shape. It should be noticed that the light intensity firstly keeps constant for a while $(\sim 2 \mathrm{~s})$ after removing the voltage, then it starts to decrease. This result implies that the expanded surface of the droplet occupies a large area, and it takes two seconds for the droplet to deflect light.

Like previous liquid lenses, the response time of our liquid lens is dependent on the amplitude of the voltage pulse. Figure 8(b) shows the response time versus the applied voltage. When the amplitude of the voltage pulse increases, the $\tau_{\text {rise }}\left(\tau_{\text {decay }}\right)$ has a tendency to decrease (increase). A higher voltage provides a stronger dielectric force, leading to a faster shape deformation. To recover to its original shape, a larger deformed droplet needs a longer distance to travel, leading to a slower response time $\left(\tau_{\text {decay }}\right)$.

In contrast to previous liquid lenses, the measured response time of our liquid lens is relatively slow. The main reason is due to the large diameter of the droplet aperture. Usually a slight focus change will need a short response time. For example, the focal length of a normal human eye is $f_{1}=\sim 25 \mathrm{~mm}$ when it focuses a distant object. When the eye looks at an object up close $(\sim 25 \mathrm{~cm})$, the focal length is $f_{2}=\sim 22.7 \mathrm{~mm}$. A small focal length change $(\Delta f=2.3 \mathrm{~mm})$ leads to a large dynamic imaging. As a comparison, a slight focus change of our lens (figure 7) will not need a large response time (figure 8). For a limited focus change, the response time of our lens is reasonable for the dynamic imaging.

Similar to previous liquid lenses, the size of our liquid lens is insensitive to the driving voltage because of in-plane fringing field actuation. Therefore, the size of the liquid lens is scalable. The driving voltage can be further reduced by optimizing the ITO pattern: decrease the gap of adjacent ITO stripes and increase the number of ITO stripes. Both liquids have matched density, so the gravity effect on distorting the shape of the droplet is negligible. Moreover, the droplet can be fixed in the Teflon hole due to adhesive force. The droplet will not shift when the lens cell is placed vertically. Our lens can present good mechanical stability without the issues of shaking, shocking or vibrating.

It is clear that one can easily prepare a converging lens if the optical oil is used as the droplet and glycerol is used as the surrounding medium. Different from a diverging lens, a converging lens with the same aperture size as that of a diverging lens usually can focus an object with a high resolution image. Owing to the radial interdigitated electrode, the generated fringing field can effectively deform the shape of a liquid droplet. If the electrode structure (the gap of the adjacent stripes and the number of the ITO stripes) is optimized, the driving voltage of our liquid lens can be reduced largely.

\section{Conclusion}

We have reported a dielectric liquid lens with a radial-interdigitated electrode. The newly patterned electrode can provide a symmetrical-inhomogeneous fringing field. Different from previous approaches, the generated dielectric force exerting on the surface of our liquid lens is in radial direction. Therefore, the radial-interdigitated electrode can efficiently deform the shape of a dielectric liquid lens, while the lens cell remains the merits of simple fabrication, compact structure, easy operation, and good mechanical stability. Using a $4 \mathrm{~mm}$ aperture glycerol droplet as the liquid lens and optical oil (SL5267) as the surrounding medium, its focal length can be changed from $\sim-4.41$ to $\sim-8.28 \mathrm{~mm}$ when the applied voltage is varied from 0 to $120 \mathrm{~V}_{\text {rms }}$. The demonstrated liquid lens exhibits good optical performance, and the response time is reasonable fast when its shape is deformed slightly. By optimizing the ITO electrode structure, both driving voltage and power consumption can be reduced largely. In contrast to previous approaches, our liquid lens with radial interdigitated electrode remains the advantages of scalable aperture size and wide variability of focal length. Moreover, the driving voltage is insensitive to the size of the liquid lens. Our liquid lens with radial-interdigitated electrode has promising applications in imaging processing, optical communication, machine vision, and cell phone.

\section{Acknowledgments}

The authors appreciate the financial support by the National Research Foundation of Korea under Grant 2014001345 and partially support by the Basic Science Research Program of NRF under Grant 2013063265. 


\section{References}

[1] Chronis N, Liu G L, Jeong K H and Lee L P 2003 Opt. Express 112370

[2] Zhang D Y, Lien V, Berdichevsky Y, Choi J and Lo Y H 2003 Appl. Phys. Lett. 823171

[3] Ren H, Fox D, Anderson P A, Wu B and Wu S T 2006 opt. Express 148031

[4] Oku H and Ishikawa M 2009 Appl. Phys. Lett. 94221108

[5] Dong L, Agarwal A K, Beebe D J and Jiang H 2007 Adv. Mater. 19401

[6] Koyama D, Isago R and Nakamura K 2010 Opt. Express 18 25158

[7] Xiao W and Hardt S 2010 J. Micromech. Microeng. 20055032

[8] Cheng H C, Xu S, Liu Y, Levi S and Wu S T 2011 Opt. Commun. 2842118

[9] Malouin B A Jr, Vogel M J, Olles J D, Cheng L and Hirsa A H 2011 Lab. Chip 11393

[10] Choi J M, Son H M and Lee Y J 2009 Opt. Express 178152

[11] Wei K, Domicone N W and Zhao Y 2014 Opt. Lett. 391318

[12] Shian S, Diebold R M and Clarke D R 2013 Opt. Express 218669

[13] Ren L, Lee R H, Park H R, Ren H, Nah C and Yoo I S 2013 J. Microelectromech. Syst. 221222

[14] Berge B and Peseux J 2000 Eur. Phys. J. E 3159

[15] Krupenkin T, Yang S and Mach P 2003 Appl. Phys. Lett. 82316

[16] Jones T B, Gunji M, Washizu M and Feldman M J 2001 J. Appl. Phys. 891441
[17] Kuiper S and Hendriks B H W 2004 Appl. Phys. Lett. 851128

[18] Grilli S, Miccio L, Vespini V, Finizio A, De Nicola S and Ferraro P 2008 Opt. Express 168084

[19] Lin Y H, Li J K, Chu T Y and Hsu H K 2010 Opt. Express 18 10104

[20] Ren H, Xu S and Wu S T 2011 Lab. Chip 113426

[21] McHale G, Brown C V, Newton M I, Wells G G and Sampara N 2011 Phys. Rev. Lett. 107186101

[22] Cheng C C and Yeh J A 2007 Opt. Express 157140

[23] Ren H, Xianyu H, Xu S and Wu S T 2008 Opt. Express 16 14954

[24] Hung K Y, Fan C C, Tseng F G and Chen Y K 2010 Opt. Express 186014

[25] Yang C C, Tasi C W G and Yeh J A 2011 J. Microelectromech. Syst. 201143

[26] Xu S, Ren H, Liu Y and Wu S T 2011 J. Microelectromech. Syst. 20297

[27] Lu Y S, Tu H, Xu Y and Jiang H 2013 Appl. Phys. Lett. 103 26113

[28] Demierre N, Braschler T, Linderholm P, Seger U, van Lintel H and Renaud P 2007 Lab. Chip 7355

[29] Park K, Suk H J, Akin D and Bashir R 2009 Lab. Chip 92224

[30] Penfield P and Haus H A 1967 Electrodynamics of Moving Media (Cambridge, MA: MIT press)

[31] Xu S, Ren H, Liu Y and Wu S T 2012 J. Disp. Technol. 8336

[32] Smith W J 2000 Modern Optical Engineering (New York: McGraw-Hill)

[33] Hecht E 2002 Optics (New York: Addison-Wesley) 